\title{
Online Identification of Lithium-ion Battery Model Parameters with Initial Value Uncertainty and Measurement Noise
}

\section{Xinghao Du}

Sichuan University - Wangjiang Campus: Sichuan University

Jinhao Meng ( $\square$ jinhao@scu.edu.cn )

Sichuan University - Wangjiang Campus: Sichuan University https://orcid.org/0000-0003-3490-5089

Kailong Liu

University of Warwick

Yingmin Zhang

Sichuan University - Wangjiang Campus: Sichuan University

\section{Shunli Wang}

Southwest University of Science and Technology

Jichang Peng

Nanjing Institute of Technology

Tianqi Liu

Sichuan University - Wangjiang Campus: Sichuan University

\section{Original Article}

Keywords: Li-ion battery, Equivalent circuit model, Recursive least squares, Recursive total least squares

Posted Date: September 28th, 2021

DOl: https://doi.org/10.21203/rs.3.rs-927275/v1

License: (c) (i) This work is licensed under a Creative Commons Attribution 4.0 International License.

Read Full License 


\title{
EVS34/ICEIV2021 Recommended Paper Paper Number: 3990365
}

\section{Title page}

\author{
Online Identification of Lithium-ion Battery Model Parameters \\ with Initial Value Uncertainty and Measurement Noise
}

Xing-Hao Du, born in 1998, is currently pursuing the M.S. degree with the College of Electrical Engineering, Sichuan University, China. He received his bachelor degree from Civil Aviation Flight University of China, Deyang, China, in 2020. His research interests include lithium-ion batteries modeling, state-of-charge estimation and battery management systems.

Tel: +86-136-18087804; E-mail: duxinghao@stu.scu.edu.cn

\begin{abstract}
Jin-Hao Meng, born in 1988, is currently an associate researcher in Sichuan University, Chengdu, China. He received the M.S. degree in control theory and control engineering and the Ph.D. degree in electrical engineering from Northwestern Polytechnical University (NPU), Xi'an, China, in 2013 and 2019, respectively.
\end{abstract}

E-mail: jinhao@scu.edu.cn

Kai-Long Liu, is currently is a Research Fellow with the Warwick Manufacturing Group, University of Warwick, U.K. He received the B.Eng. degree in electrical engineering and the M.Sc. degree in control theory and control engineering from Shanghai University, China, in 2011 and 2014, respectively, and the Ph.D. degree in electrical engineering from the Energy, Power and Intelligent Control Group, Queen's University Belfast, Belfast, U.K., in 2018.

E-mail: kliu02@qub.ac.uk

Ying-Min Zhang, born in 1974, is currently a Professor with the College of Electrical Engineering, Sichuan University, China. She received the M.S. and the Ph.D. degrees in electrical engineering from Sichuan University, Chengdu, China, in 1998 and 2013, respectively. E-mail: zhangyingmin@scu.edu.cn

Shun-Li Wang, born in 1985, is currently a Professor with the School of Information Engineering, Southwest University of Science and Technology, China. He received the M.S. and the Ph.D. degrees from Southwest University of Science and Technology, China, in 2012 and 2018, respectively.

E-mail: wangshunli@swust.edu.cn

Ji-Chang Peng, born in 1988, is currently a lecturer in Nanjing Institute of Technology, Nanjing, China. He received the B.S. degree in electrical engineering from North China University of Water Resources and Electric Power, China, in 2010, and the M.S. degree in control theory and control engineering and the Ph.D. degree in electrical engineering, both from Northwestern Polytechnical University, China, in 2013 and 2019, respectively.

E-mail: linkpjc@gmail.com

Tian-Qi Liu, born in 1962, is currently a Professor with the College of Electrical Engineering, Sichuan University, China. She received the B.S. and the M.S. degrees from Sichuan University, China, in 1982 and 1986, respectively, and the Ph.D. degree from Chongqing University, China, in 1996, all in electrical engineering.

E-mail: tqliu@scu.edu.cn

Corresponding author: Jin-Hao Meng E-mail: jinhao@scu.edu.cn 


\title{
Online Identification of Lithium-ion Battery Model Parameters with Initial Value Uncertainty and Measurement Noise
}

\author{
Xing-Hao Du ${ }^{1} \bullet$ Jin-Hao Meng ${ }^{1} \bullet$ Kai-Long Liu $^{2} \bullet$ Ying-Min Zhang ${ }^{1} \bullet$ Shun-Li Wang ${ }^{3} \bullet$ Ji-Chang Peng ${ }^{4} \cdot$ Tian-Qi \\ Liu ${ }^{1}$
}

\begin{abstract}
Online parameter identification is essential for the accuracy of the battery Equivalent Circuit Model (ECM). The traditional Recursive Least Squares (RLS) method is easily biased with the noise disturbances from sensors, which degrades the modeling accuracy in practice. Meanwhile, the Recursive Total Least Squares (RTLS) method can deal with the noise interferences, but the parameter slowly converges to the reference with initial value uncertainty. To alleviate the above issues, this paper proposes a co-estimation framework utilizing the advantages of RLS and RTLS for a higher parameter identification performance of the battery ECM. RLS converges quickly by updating the parameters along the gradient of the cost function. RTLS is applied to attenuate the noise effect once the parameters have converged. Both simulation and experimental results prove that the proposed method has good accuracy, fast convergence rate, and also robustness against noise corruption.
\end{abstract}

Keywords: Li-ion battery $\bullet$ Equivalent circuit model $\bullet$ Recursive least squares $\bullet$ Recursive total least squares

\section{Introduction}

Lithium-ion (Li-ion) batteries are widely used in Electric Vehicles (EVs) and stationary energy storage because of their high charge/discharge efficiency, low self-discharge rate, and long lifespan [1]-[3]. To extend the service life of the batteries and ensure their safe operation, a well-designed Battery Management System (BMS) is required to monitor the State of Health (SOH) and State of Charge (SOC) [4]-

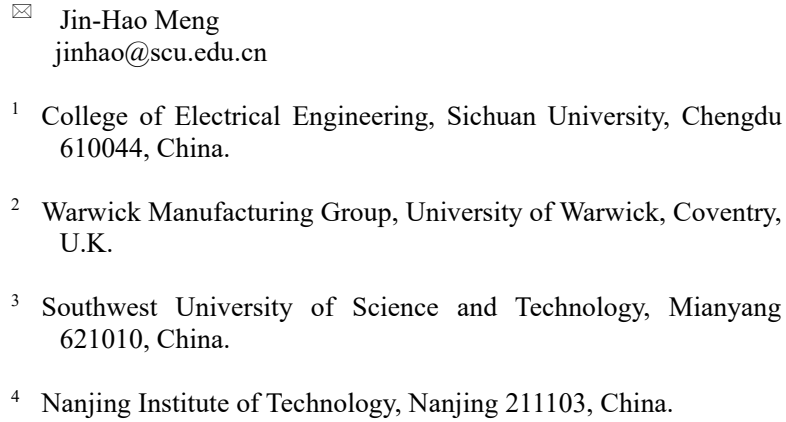

4 Nanjing Institute of Technology, Nanjing 211103, China.

[6]. Model-based estimation approaches, such as Kalman filters and particle filter, have been proposed to realize these functionalities. The model-based methods generally require an accurate battery model to ensure their performance [7].

The commonly used battery models include electrochemical models and ECMs. Electrochemical models describe the partial differential equations of the electrochemical reactions inside the battery [8]. As such, great efforts are required on parameterization and dealing with the computational burden. In contrast, ECMs only use RC (Resistance and Capacitance) elements to express the external characteristics of the batteries, which can balance the contradiction between the modeling accuracy and the complexity [9]. In this way, ECMs are considered more suitable for online states estimation of the batteries in a BMS [10].

The characteristics of the Li-ion battery usually change with external factors such as temperature, current rate, and aging, etc. It is easily realized that the RC parameters in the battery ECM vary with those external factors in real applications [11]. Thus, the suitability and accuracy of ECMs for a specific battery are closely related to the parameter identification method. If the parameters of ECM deviate from the reasonable ranges, the performance of the battery model will be questionable [12]. Both offline and online methods can be used for parameter identification of the ECM. Offline parameter identification methods require sufficient laboratorial labor, in order to collect enough measurement data for parameter extraction [13]. But we cannot test the Liion battery covering all its working conditions. Online methods are not high relying on the additional tests, which can identify the parameters of a battery ECM from the current and voltage measurement of the sensors. In this regard, a large amount of online parameter identification methods has been proposed in the literature, which can be briefly divided into nonlinear filter based methods [14], [15] and Least-Squares (LS) [7], [16]-[19] based methods. Nonlinear filters, such as extended Kalman filters, normally need to tune the covariance matrixes for an acceptable 
accuracy, which are difficult to be adjusted in real-time applications. LS-based methods have the advantages of easy tuning and a lower computational cost [18] and are further investigated in this paper.

RLS is the most widely used method for online parameter identification. Xiong et al. [16] employ the RLS method to track the real-time characteristics of $32 \mathrm{Ah} \mathrm{Li-ion} \mathrm{batteries.}$ Many efforts have been found to improve the accuracy of RLS. Duong et al. [17] propose a Multiple Adaptive Forgetting Factors based RLS (MAFF-RLS) method to capture the variations and different dynamics of the parameters in ECM. Ouyang et al. [7] use a robust RLS algorithm coping with the outliers of battery measurement.

One drawback of the RLS-based methods is that they are sensitive to measurement noises. Unexpected noises always exist and cannot be eliminated easily, which inevitably leads to biased parameter identification results in practice. As an alternative choice, the Total Least Square (TLS) method can effectively deal with the measurement noises from sensors [20]. Wei et al. [21], [22] has employed an RQ-based RTLS method for the online parameter identification of the ECM, which alleviates the model identification bias caused by noise disturbances. Although the method shows good accuracy and robustness against noise corruption, the convergence speed has not been fairly discussed in their work. Considering the parameters of the battery model change with the battery stats, an ideal parameter identification method should have a good accuracy as well as a superior convergence speed.

Regarding convergence speed, RLS updates the parameters along the gradient of the cost function, which has a rather fast convergence rate [23]. Although the RQ-based RTLS method [20] adopts a similar form of the gradient search strategy as RLS, the convergence rate of the RTLS decreases significantly with unknown initial parameter values. It's worth to mention that the convergence rate has a great influence on whole system stability [7].

Therefore, RLS converges the parameters quickly with low computational cost, while the identification results are biased with measurement noises. Although RTLS can deal with noise corruption, the convergence speed is slow. In order to cope with the above issues, this paper proposes a coestimation method, which combines the advantages of RLS and RTLS. Simulation and experimental results confirm that the proposed method can accurately identify the parameters in the presence of measurement noises and initial value uncertainty.

The key contributions of this paper are in the following aspects.

(1) A comprehensive study is constructed to analyze the advantages and deficiencies of RLS and RTLS for online parameter identification.

(2) Combining the merits of RLS and RTLS, a coestimation method is designed, which owns fast convergence speed with initial value uncertainty and robustness against noise interferences.

(3) The proposed method is validated under dynamic driving cycles in both simulation and experimental tests comparing with RLS and RTLS.

The remainder of this paper is organized as follows. Section 2 introduces the modeling strategy for the battery. Section 3 compares the performances of RLS and RTLS, and presents the motivation of the proposed co-estimation method. Simulation and experimental results are carried out in Sections 4 and 5, respectively. The main conclusions are given in Section 6.

\section{Battery Modeling}

Considering a balance of modeling accuracy and simplicity, ECM is preferred and investigated in this paper. Among all the ECMs, the Thevenin model has a relatively simple structure, which can capture the primary dynamics of the battery without taking much computing resources.

As shown in Figure 1, the Thevenin model consists of a series resistor and a parallel RC network. $R_{0}$ represents the Ohmic resistance, which is used to describe the instantaneous voltage drop when a current excitation is applied to the battery. The RC network aims at describing dynamic characteristics of the battery, such as kinetic effect and ion diffusing.

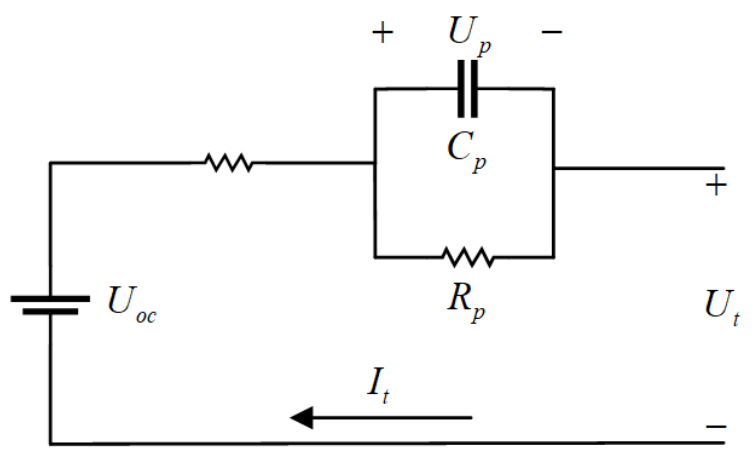

Figure 1 Circuit diagram of the Thevenin model.

The battery Open Circuit Voltage (OCV) $U_{o c}$ is expressed as,

$$
U_{\mathrm{oc}}(S)=\sum_{i=0}^{m} k_{i} S^{i}
$$


where $S$ is the battery SOC, $k_{i}(i=1,2, \ldots, m)$ are the polynomial coefficient of the OCV-SOC curve, $m$ is the order of the function.

The terminal voltage and current of the battery are expressed as $U_{t}$ and $I_{t}$, respectively. The transfer function of the Thevenin model is established as,

$$
G(s)=\frac{U_{O C}(s)-U_{t}(s)}{I_{t}(s)}=R_{0}+\frac{R_{p}}{1+s R_{p} C_{p}}
$$

where $s$ is the Laplace operator.

By applying the bilinear transform in (3), the discrete form of the Thevenin model in (2) can be expressed as (4).

$$
\begin{array}{r}
s=\frac{2\left(z^{-1}-1\right)}{T_{s}\left(z^{-1}+1\right)} \\
G\left(z^{-1}\right)=\frac{b_{0}+b_{1} z^{-1}}{1+a_{1} z^{-1}}
\end{array}
$$

where $z$ is the discretization operator, $a_{1}, b_{1}$, and $b_{2}$ are the coefficients defined as (5).

$$
\left\{\begin{array}{l}
a_{1}=\frac{T_{s}-2 R_{p} C_{p}}{T_{s}+2 R_{p} C_{p}} \\
b_{0}=-\frac{R_{0} T_{s}+R_{p} T_{s}+2 R_{p} C_{p} R_{0}}{T_{s}+R_{p} C_{p}} \\
b_{1}=-\frac{R_{0} T_{s}+R_{p} T_{s}-2 R_{p} C_{p} R_{0}}{T_{s}+R_{p} C_{p}}
\end{array}\right.
$$

where $T_{s}$ is the sampling interval.

Applying the linear regression method, Eq. (4) can be rewritten as a linear equation,

$$
y_{k}=\boldsymbol{\theta}_{k}^{T} \mathbf{x}_{k}
$$

In (6), the output $y_{k}$, the estimated parameters $\boldsymbol{\theta}_{k}$, and the input $\mathbf{x}_{k}$ at time $k$ are defined as,

$$
\left\{\begin{array}{l}
y_{k}=U_{L, k} \\
\boldsymbol{\theta}_{k}=\left[b_{0, k}, b_{1, k}, a_{1, k}\right]^{T} \\
\mathbf{x}_{k}=\left[I_{k}, I_{k-1}, U_{L, k-1}\right]^{T}
\end{array}\right.
$$

Once $\boldsymbol{\theta}_{k}$ is obtained, the parameters of the Thevenin model can be deduced as,

$$
\left\{\begin{array}{l}
R_{0, k}=\frac{b_{2, k}-b_{1, k}}{1-a_{1, k}} \\
R_{p, k}=\frac{2\left(a_{1, k} b_{1, k}-b_{2, k}\right)}{1-a_{1, k}^{2}} \\
C_{p, k}=\frac{T_{s}\left(1-a_{1, k}^{2}\right)^{2}}{4\left(a_{1, k} b_{1, k}-b_{2, k}\right)}
\end{array}\right.
$$

\section{Online Parameter Identification}

\subsection{Least Squares}

LS performs the parameter identification by minimizing the squares of the errors between the terminal voltage and the output of the battery model [24]. As shown in Figure 2, LS assumes that the measured output $\tilde{y}$ is noisy while the input $x$ is accurate.

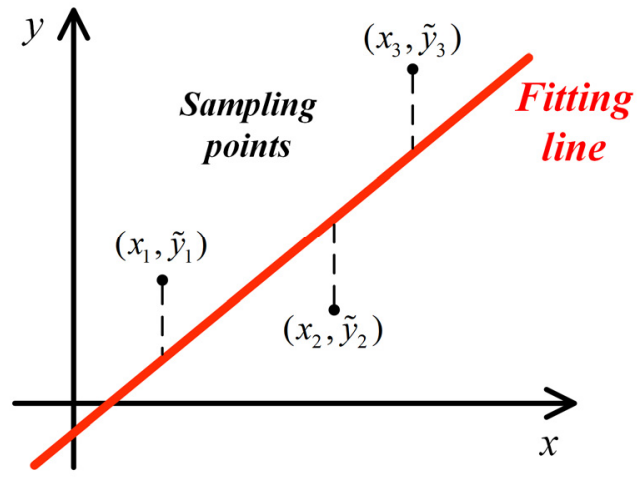

Figure 2 The principle of the LS method.

For LS, the parameter vector $\boldsymbol{\theta}_{k}$ can be solved by minimizing the cost function as follows,

$$
J\left(\boldsymbol{\theta}_{k}\right)=\sum_{i=1}^{k}\left[\Delta y_{i}\right]^{2}=\sum_{i=1}^{k}\left[\tilde{y}_{i}-\boldsymbol{\theta}_{k}^{T} \mathbf{x}_{i}\right]^{2}
$$

where $\Delta y_{i}$ is the measurement error, $\tilde{y}_{i}$ is the noisy output. Defined that the gradient of the cost function $J\left(\boldsymbol{\theta}_{k}\right)$ is equal to zero,

$$
\partial J\left(\boldsymbol{\theta}_{k}\right) / \partial \boldsymbol{\theta}_{k}=0
$$

Then, the analytical solution of the parameter vector $\boldsymbol{\theta}_{k}$ can be obtained as,

$$
\boldsymbol{\theta}_{k}=\left(\mathbf{X}_{k}^{T} \mathbf{X}_{k}\right)^{-1} \mathbf{X}_{k}^{T} \mathbf{Y}_{k}
$$

where $\mathbf{X}_{k}=\left[\mathbf{x}_{1}, \mathbf{x}_{2}, \ldots, \mathbf{x}_{k}\right]^{T}, \mathbf{Y}_{k}=\left[y_{1}, y_{2}, \ldots, y_{k}\right]^{T}$.

The recursive form of the LS can be further expressed as,

$$
\left\{\begin{array}{l}
\mathbf{K}_{k}=\mathbf{P}_{k-1} \mathbf{x}_{k} /\left(\lambda+\mathbf{x}_{k}^{T} \mathbf{P}_{k-1} \mathbf{x}_{k}\right) \\
e_{k}=y_{k}-\mathbf{x}_{k}^{T} \hat{\boldsymbol{\theta}}_{k-1} \\
\boldsymbol{\theta}_{k}=\boldsymbol{\theta}_{k-1}+\mathbf{K}_{k} e_{k} \\
\mathbf{P}_{k}=\left(\mathbf{I}-\mathbf{K}_{k} \mathbf{x}_{k}^{T}\right) \mathbf{P}_{k-1} / \lambda
\end{array}\right.
$$


where $\mathbf{K}_{k}$ denotes the gain matrix, $\mathbf{P}_{k}$ is the covariance matrix, $e_{k}$ is the residual error, and $\lambda(0.95<\lambda<1)$ is a user-defined forgetting factor.

It should be noted that LS has not considered the errors from the input $\mathbf{x}$. Thus, the estimation results are easily biased owing to the noise corruption.

\subsection{Total Least Squares}

Different from LS, TLS assumes that both output $\tilde{y}$ and input $\tilde{x}$ are noisy. As we can see from Figure 3, TLS employs the orthogonal regression to minimize the sum of the squared orthogonal distances from the sampling points to the fitting line.

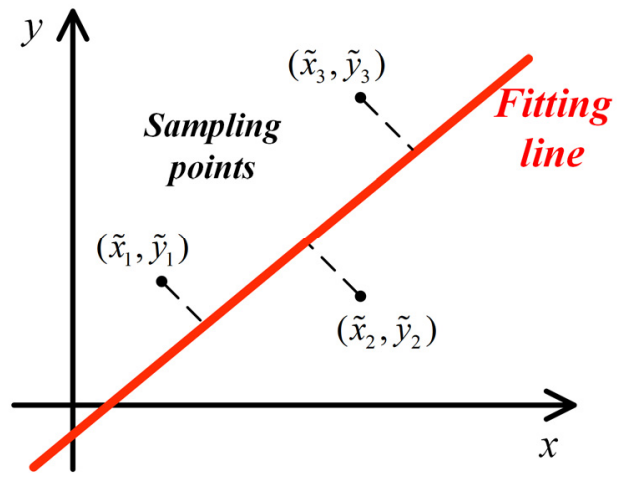

Figure 3 The principle of the TLS method.

Similarly, TLS solves the parameter vector $\boldsymbol{\theta}_{k}$ by minimizing the cost function as follows,

$$
J\left(\boldsymbol{\theta}_{k}\right)=\left\|\left[\Delta \mathbf{X}_{k}, \Delta \mathbf{Y}_{k}\right]\right\|_{F}
$$

where $\Delta \mathbf{X}_{k}=\left[\Delta \mathbf{x}_{1}, \Delta \mathbf{x}_{2}, \ldots, \Delta \mathbf{x}_{k}\right]^{T}, \Delta \mathbf{Y}_{k}=\left[\Delta y_{1}, \Delta y_{2}, \ldots, \Delta y_{k}\right]^{T}$.

The recursive form of the TLS is expressed as,

$$
\boldsymbol{\theta}_{k}=\boldsymbol{\theta}_{k-1}+\alpha_{k} \tilde{\mathbf{x}}_{k}
$$

where the gain factor $\alpha_{k}$ is obtained by using the gradient search approach in [21],

$$
\partial J\left(\boldsymbol{\theta}_{k-1}+\alpha_{k} \tilde{\mathbf{x}}_{k}\right) / \partial \alpha_{k}=0
$$

where $\tilde{\mathbf{x}}_{k}$ is the noisy input vector.

As shown in (14), RTLS updates the parameters along the direction of $\tilde{\mathbf{x}}_{k}$ rather than the gradient of the cost function. Only one gain factor $\alpha_{k}$ can be obtained at each iteration, which largely limits the convergence rate when multiple parameters are needed to be identified.

\subsection{A Comparison between RLS and RTLS}

A comparative study is carried out in this subsection to evaluate the performances of RLS and RTLS for online parameter identification.

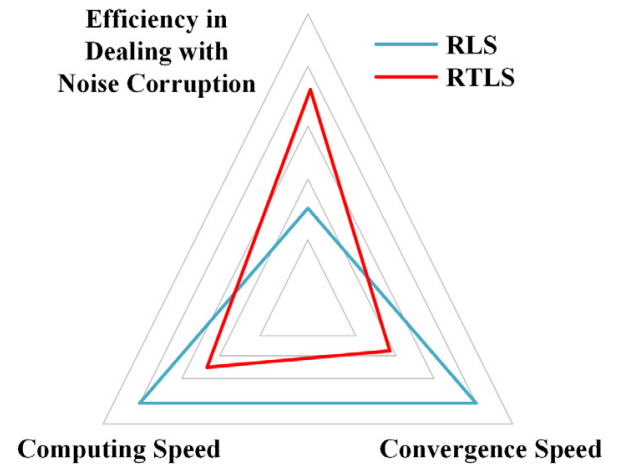

Figure 4 Performance comparison between RLS and RTLS

As shown in Figure 4, RLS and RTLS have some merits from a specific area. On one hand, RTLS takes into account the disturbances from both the input and output, which has a better performance in dealing with noise interferences. On the other hand, RLS updates the parameters along the gradient of the cost function, which owns a very fast computing speed and a higher convergence rate.

However, RLS is biased with measurement noises, while RTLS converges slowly with initial value uncertainty. Therefore, to design a superior approach dealing with the above issues, this paper integrates the RLS and RTLS for better parameter identification of the ECM.

\subsection{The Proposed Co-estimation Method}

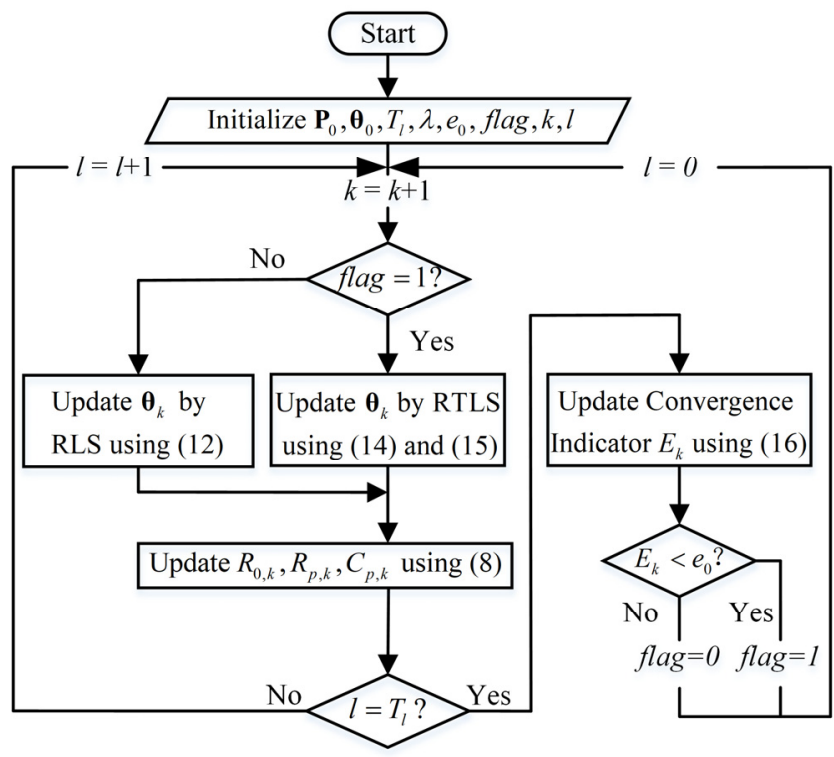

Figure 5 Flowchart of the proposed parameter identification method

This work proposes a co-estimation algorithm for superior performances of online parameter identification, which has fast convergence speed and robustness against noise 
corruption. Meanwhile, the proposed method does not require much computational effort and is suitable for online implementation. The flowchart of the proposed method is as follows.

The variables in Figure 5 are described as follows. (i) $e_{i}(i$ $\left.=k-T_{L}, k-T_{L}+1, \ldots, k\right)$ is the residual error of the parameter identification. (ii) $e_{0}$ is set as a threshold to decide the convergence of the parameters. (iii) $T_{L}$ is the time scale for determining the convergence of the parameters. (iv) $E_{k}$ is designed as a convergence indicator, which is expressed as the Root Mean Square Error (RMSE) of $e_{i}$,

$$
E_{k}=\sqrt{\frac{1}{T_{l}} \sum_{i=k-T_{l}}^{k} e_{i}^{2}}
$$

The strategy of the proposed co-estimation method can be summarized as the following three parts.

Part I. Given the initial parameter values are unavailable, $\boldsymbol{\theta}_{k}$ is randomly initialized and updated by RLS using (12) until the parameters are converged to their references.

Part II. To determine whether the parameters have converged, $E_{k}$ is calculated using (16) once $l$ reaches $T_{L}$.

Part III. When $E_{k}$ is less than the pre-set threshold $e_{0}$, the flag is set to 1 , indicating the convergence has completed. The parameters are updated by RTLS using (14) and (15) afterwards.

One can see the proposed co-estimation method combines the merits of RLS and RTLS. RLS can converge to the reference values of the parameters quickly without any prior knowledge on initial values, while RTLS has good accuracy and robustness against the noise disturbances, which can be applied to update the already converged parameters.

\section{Simulation Validation}

A simulated battery model is used in this section to verify the performance of the proposed co-estimation method. It is noteworthy that the Ohmic resistance $R_{0}$ is stable during the discharging process while $R_{p}$ and $C_{p}$ tend to vary with SOC and current rate [25]. Therefore, the model parameter $R_{0}$ is defined as a constant, while that of $R_{p}$ and $C_{p}$ are timevarying. The OCV is obtained by the OCV-SOC relationship as in (1). The sampling frequency of voltage and current is set to $1 \mathrm{~Hz}$.

The Urban Dynamometer Driving Schedule (UDDS) is applied to the simulated battery model. The voltage and current profiles are shown in Figure 6.

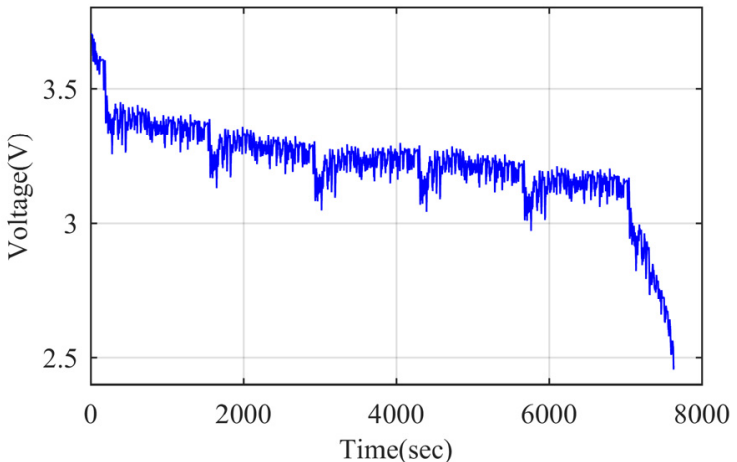

(a)

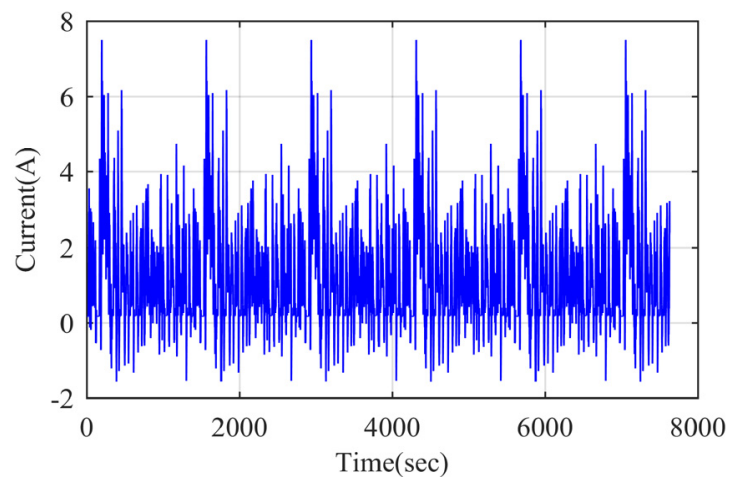

(b)

Figure 6 Voltage and current profiles in the simulation test. (a) voltage. (b) current.

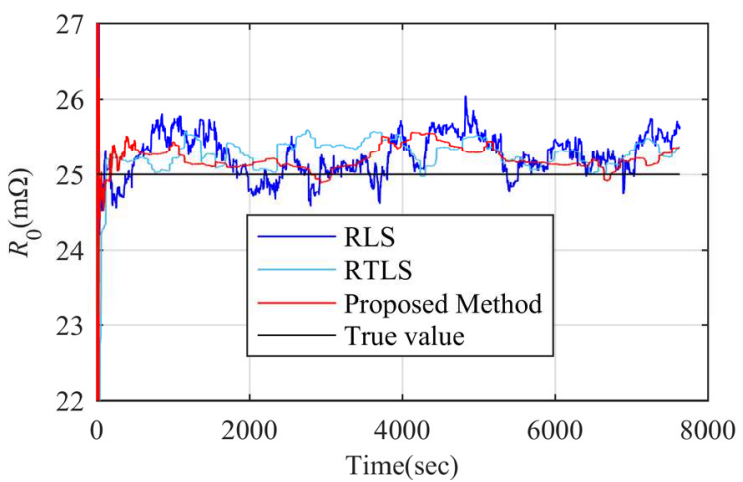

(a)

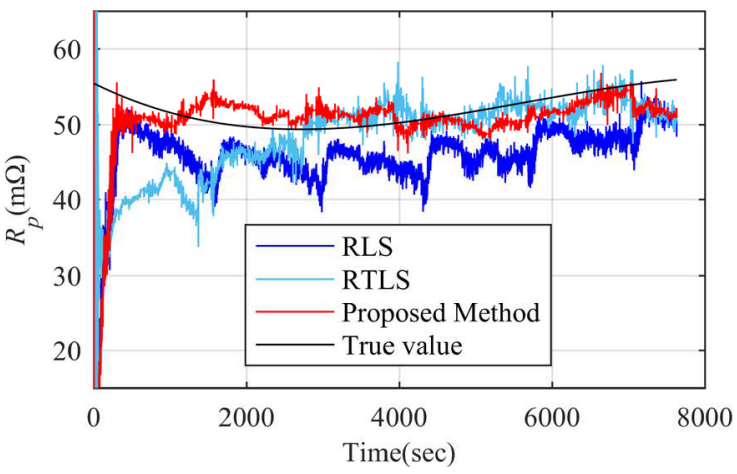

(b) 


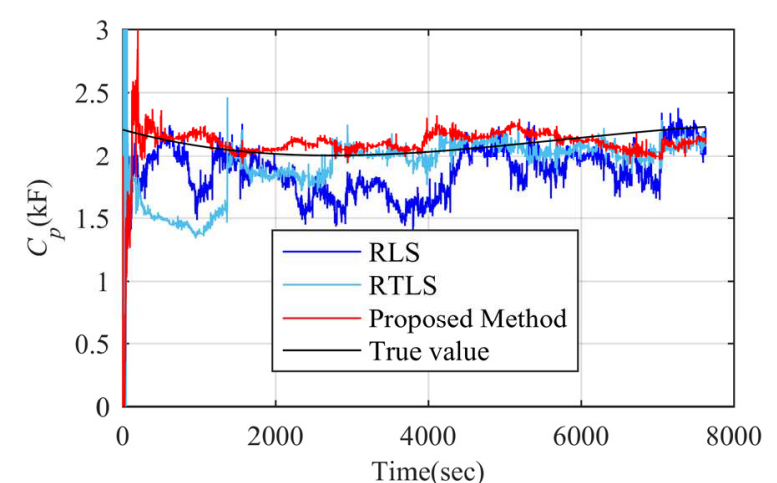

(c)

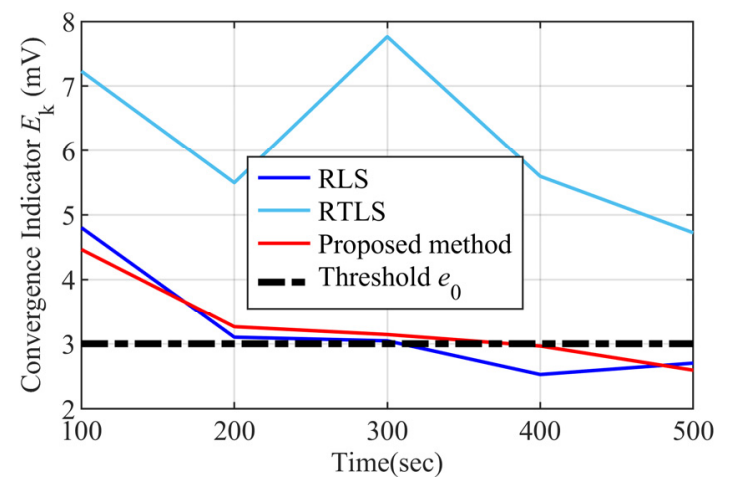

(d)

Figure 7 Simulation results of the model parameters. (a) $R_{0}$. (b) $R_{p}$. (c) $C_{p}$. (d) $E_{k}$

The unknown initial values of the model parameters are randomly initialized as $R_{0}=20 \mathrm{~m} \Omega, R_{p}=20 \mathrm{~m} \Omega, C_{p}=1000$ $F$. Besides, $T_{L}$ and $e_{0}$ in the proposed method are initialized as $100 \mathrm{~s}$ and $3 \mathrm{mV}$, respectively. To verify the performance of the proposed method with noise interference, white Gaussian noises are randomly added to the voltage and current measurements. The Standard Deviations (SDs) $\sigma_{v}$ and $\sigma_{i}$ are set to $4 \mathrm{mV}$ and $4 \mathrm{~mA}$ in this validation.

As shown in Figure 7, the estimation of $R_{0}$ is not sensitive to the measurement noises, where the estimated values are close to the true values for all three methods. Regarding $R_{p}$ and $C_{p}$, the estimated values of RLS are biased from the true values owing to the noise disturbances. As for RTLS, it takes a long time for the parameters to converge, which greatly enlarges the estimation error of the model.

It can be seen from Figure 7 (d), the proposed method has the same convergence speed as RLS. Once $E_{k}$ is less than $e_{0}$, the converged parameters are further updated by RTLS in dealing with the noise interferences. Thanks to the welldesigned parameter updates mechanism, the proposed method shows a good estimation accuracy and robustness, where the estimated values can well track the true values almost all the time.
To quantitively evaluate the estimation accuracy of the model parameters, the Mean Square Deviation (MSD) is selected as,

$$
\mathrm{MSD}=10 \log _{10}\left(E\left[\left\|\mathbf{e}_{k}\right\|_{2}^{2}\right]\right)
$$

where $\mathbf{e}_{k}$ is the normalized error expressed as,

$$
\mathbf{e}_{k}=\left[\frac{\Delta R_{0, k}}{R_{0, k}}, \frac{\Delta R_{p, k}}{R_{p, k}}, \frac{\Delta C_{p, k}}{C_{p, k}}\right]
$$

where $\Delta R_{0, k}, \Delta R_{p, k}$, and $\Delta C_{p, k}$ are the errors between the estimated parameters and the true values at the time step $k$. The average MSDs of all three methods are presented in Table 1 .

Table 1. Average MSDs of Different Methods

\begin{tabular}{cccc}
\hline & RLS & RTLS & $\begin{array}{c}\text { Proposed } \\
\text { method }\end{array}$ \\
\hline Average MSD & $-13.05 \mathrm{~dB}$ & $-15.58 \mathrm{~dB}$ & $-17.07 \mathrm{~dB}$ \\
\hline
\end{tabular}

As we can see the average MSD of the proposed method is merely $-17.07 \mathrm{~dB}$, which represents a higher accuracy of parameter identification under noise interference and initial value uncertainty. The above results coincide with the theoretical analysis in Section 4, the effectiveness of the proposed method is then proved by a battery simulation model.

\section{Experimental Validation}

Experimental tests are carried out on a $\mathrm{LiFePO}_{4}$ battery to validate the proposed method in this subsection. The specifications of the battery are listed in Table 2. The OCVSOC polynomial coefficients are listed in Table 3.

Table 2. Specifications of the $\mathrm{LiFePO}_{4}$ Battery

\begin{tabular}{ccccc}
\hline Model & $\begin{array}{c}\text { Nominal } \\
\text { Capacity } \\
\text { (Ah) }\end{array}$ & $\begin{array}{c}\text { Nominal } \\
\text { voltage(V) }\end{array}$ & $\begin{array}{c}\text { Charge } \\
\text { Cut-off } \\
\text { voltage(V) }\end{array}$ & $\begin{array}{c}\text { Discharge } \\
\text { Cut-off } \\
\text { voltage(V) }\end{array}$ \\
\hline ANR26650 & 2.5 & 3.2 & 3.6 & 2 \\
\hline
\end{tabular}

Table 3. Polynomial coefficients of the OCV-SOC function

\begin{tabular}{ccccc}
\hline$k_{0}$ & $k_{1}$ & $k_{2}$ & $k_{3}$ & $k_{4}$ \\
\hline 2.567 & 15.92 & -152.8 & 754.7 & -2081 \\
$k_{5}$ & $k_{6}$ & $k_{7}$ & $k_{8}$ & \\
3315 & -3012 & 1437 & -275.8 & \\
\hline
\end{tabular}

In Figure 8, the battery test platform consists of a thermal chamber to control the ambient temperature, a Chroma 17011 test station to charge and discharge the battery, a host computer to program the experiment procedure and store the measurement data. The sampling frequency is set to $1 \mathrm{~Hz}$. The ambient temperature is set to $25^{\circ} \mathrm{C}$ during the test. 


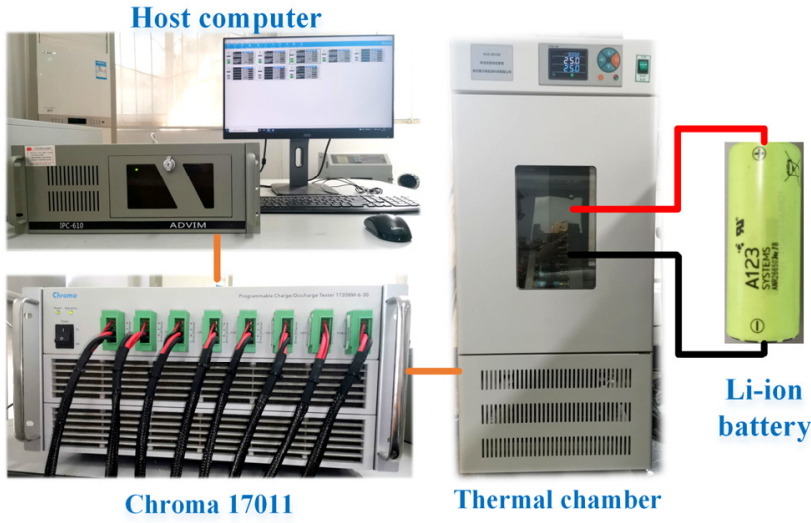

Figure 8 Experimental platform for the battery test

To verify the proposed method under noise corruption, white Gaussian noises with variances of $\sigma_{v}^{2}=8 \mathrm{mV}^{2}, \sigma_{i}^{2}=8$ $\mathrm{mA}^{2}$ are randomly added to the voltage and current measurements. As the initial values of the parameters are unknown, they are randomly initialized as $R_{0}=15 \mathrm{~m} \Omega, R_{p}=$ $35 \mathrm{~m} \Omega, C_{p}=400 F . T_{L}$ and $e_{0}$ are set as $100 \mathrm{~s}$ and $3 \mathrm{mV}$, which are the same as the simulation test.

The parameter identification results are presented in Figure 9. Similar to the simulation, the noise effect degrades the estimation accuracy of RLS, where the modeling error is larger than the other two methods. Although the RTLS can deal with the disturbances from measurement noises, the modeling error is still large before the parameters can converge to the references. As expected, the proposed method can alleviate the above issues and maintain a stable performance during the whole driving cycle. As shown in Table 4, the Mean Absolute Error (MAE) and RMSE of the proposed method are only $1.26 \mathrm{mV}$ and $2.26 \mathrm{mV}$. The advantages of the proposed method are proved by experimental tests.

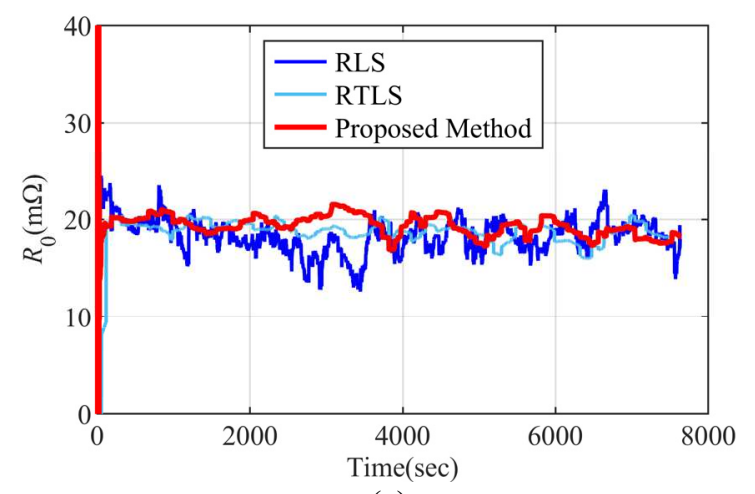

(a)

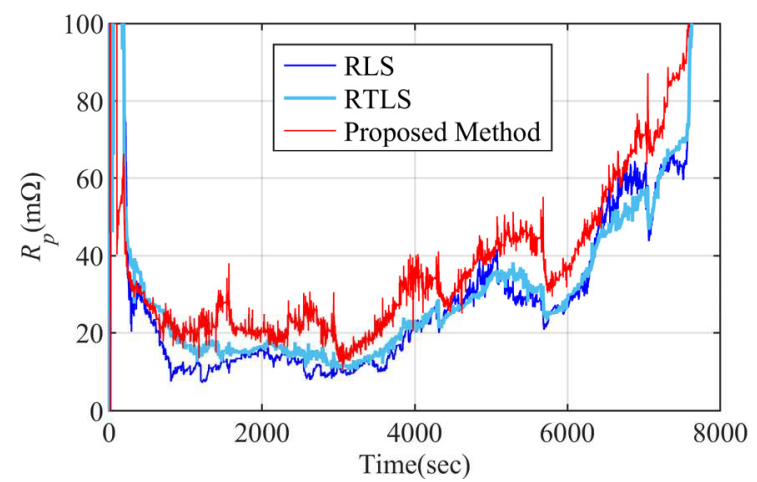

(b)

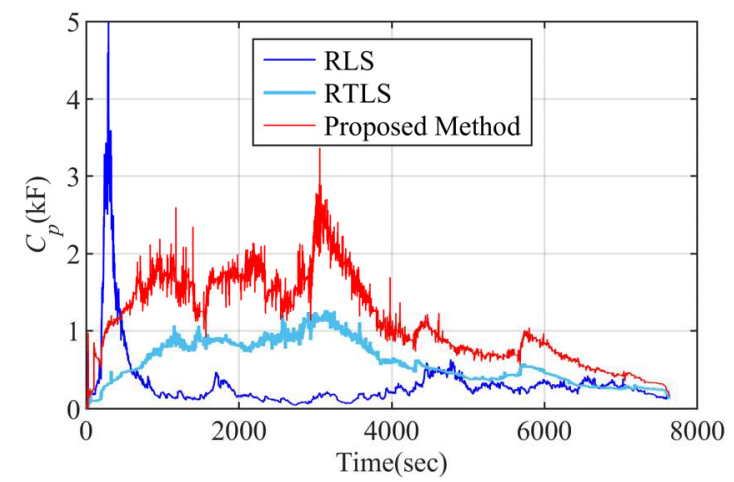

(c)

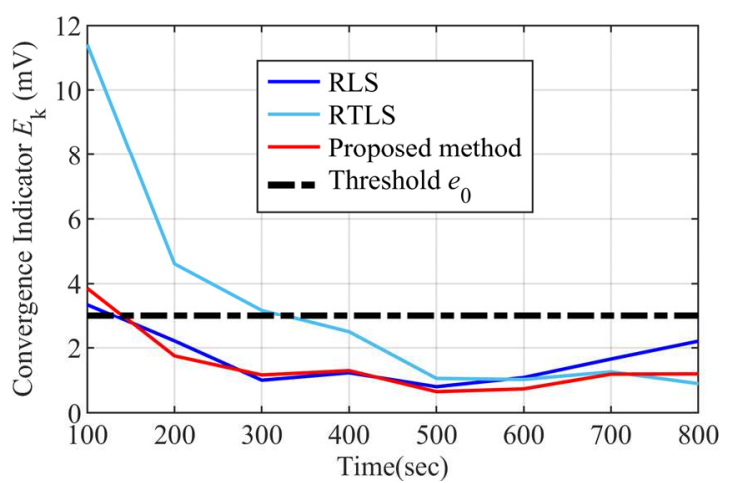

(d)

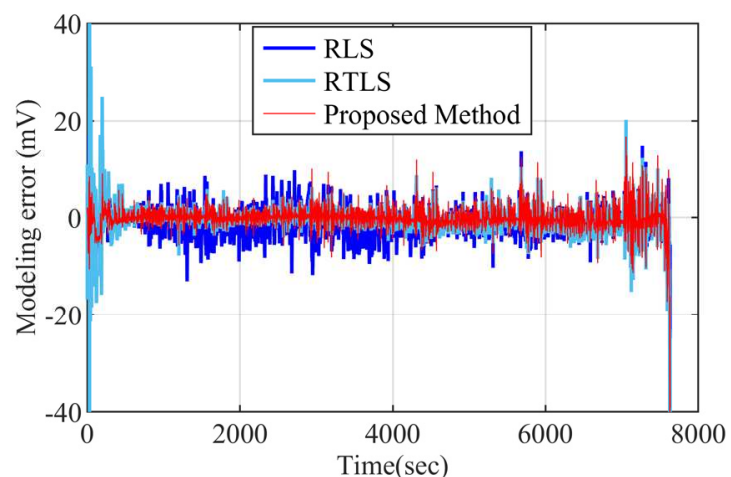

(e)

Figure 9 Experimental results of the parameter identification. (a) $R_{0}$. (b) $R_{p}$. (c) $C_{p}$. (d) $E_{k}$. (e) Modeling error. 
Table 4. MAE and RMSE of Modeling error

\begin{tabular}{cccc}
\hline & RLS & RTLS & $\begin{array}{c}\text { Proposed } \\
\text { method }\end{array}$ \\
\hline MAE $(\mathrm{mV})$ & 2.41 & 1.65 & 1.26 \\
RMSE $(\mathrm{mV})$ & 3.13 & 3.11 & 2.26 \\
\hline
\end{tabular}

\section{Conclusions}

The traditional RLS method is biased with the measurement noises from sensors, which degrades the parameter identification accuracy. The RTLS method can alleviate the noise disturbances, while the parameters converge slowly with initial value uncertainty. In this regard, we have proposed a co-estimation method, which integrates the RLS and RTLS for parameter identification. Without any prior knowledge, RLS can identify the parameters with a fast convergence rate. Once the parameters have converged, RTLS is applied to keep updating the parameters in dealing with the noise effect.

Both simulation and experimental tests have verified the validity of the proposed method in terms of convergence rate and robustness against noise corruption. The average MSD of the proposed method is merely $-17.07 \mathrm{~dB}$ in the simulation test. The MAE and RMSE of the modeling error are only $1.26 \mathrm{mV}$ and $2.26 \mathrm{mV}$ in the experimental test.

\section{Declaration}

\section{Acknowledgements \\ Not applicable}

\section{Authors' contributions}

$\mathrm{XH}-\mathrm{D}$ and JH-M conceived this study. XH-D, JH-M, and KL-L write the manuscript. YM-Z, SL-W, JC-P, and TQ-L supervised this study. All authors read and approved the final manuscript.

\section{Funding}

Supported by National Natural Science Foundation of China (Grant No. 52107229), Fundamental Research Funds for the Sichuan Science and Technology Program (Grant No. 2021YJ0063), China Postdoctoral Science Foundation (Grant No. 2020M673218), Hunan High-tech Industry Science and Technology Innovation Plan (Grant No. 2020GK2081), and the Fund of Robot Technology Used for Special Environment Key Laboratory of Sichuan Province (Grant No. 20KFKT02).

\section{Competing interests}

The authors declare no competing financial interests.

\section{Consent for publication}

Not applicable

\section{Ethics approval and consent to participate} Not applicable

\section{References}

[1] H. Dong et al., "Energy - Optimal Braking Control Using a Double - Layer Scheme for Trajectory Planning and Tracking of Connected Electric Vehicles," Chinese J. Mech. Eng., 2021, doi: 10.1186/s10033-021-00601-3.

[2] R. Du, X. Hu, S. Xie, L. Hu, Z. Zhang, and X. Lin, "Battery aging- and temperature-aware predictive energy management for hybrid electric vehicles," J. Power Sources, vol. 473, no. January, p. 228568, 2020, doi: 10.1016/j.jpowsour.2020.228568.

[3] J. Meng et al., "An Overview and Comparison of Online Implementable SOC Estimation Methods for Lithium-Ion Battery," IEEE Trans. Ind. Appl., vol. 54, no. 2, pp. 1583-1591, 2018, doi: 10.1109/TIA.2017.2775179.

[4] W. Wang, J. Wang, J. Tian, J. Lu, and R. Xiong, “Application of Digital Twin in Smart Battery Management Systems," Chinese J. Mech. Eng., 2021, doi: 10.1186/s10033-021-00577-0.

[5] J. Meng, D. I. Stroe, M. Ricco, G. Luo, and R. Teodorescu, “A simplified model-based state-of-charge estimation approach for lithium-ion battery with dynamic linear model," IEEE Trans. Ind. Electron., vol. 66, no. 10, pp. 7717-7727, 2019, doi: 10.1109/TIE.2018.2880668.

[6] H. A. Gabbar, A. M. Othman, and M. R. Abdussami, "Review of Battery Management Systems ( BMS ) Development and Industrial Standards," 2021.

[7] Q. Ouyang, J. Chen, and J. Zheng, "State-of-Charge Observer Design for Batteries with Online Model Parameter Identification: A Robust Approach," IEEE Trans. Power Electron., vol. 35, no. 6, pp. 5820-5831, 2020, doi: 10.1109/TPEL.2019.2948253.

[8] J. Tian, R. Xiong, W. Shen, and J. Wang, “A Comparative Study of Fractional Order Models on State of Charge Estimation for Lithium Ion Batteries," Chinese J. Mech. Eng., 2020, doi: 10.1186/s10033-020-00467-x.

[9] X. Lai, S. Wang, S. Ma, J. Xie, and Y. Zheng, "Parameter sensitivity analysis and simpli fi cation of equivalent circuit model for the state of charge of lithium-ion batteries," Electrochim. Acta, vol. 330, p. 135239, 2020, doi: 10.1016/j.electacta.2019.135239.

[10] R. Zhu, B. Duan, J. Zhang, Q. Zhang, and C. Zhang, "Coestimation of model parameters and state-of-charge for lithiumion batteries with recursive restricted total least squares and unscented Kalman filter," Appl. Energy, vol. 277, no. April, p. 115494, 2020, doi: 10.1016/j.apenergy.2020.115494.

[11] B. Ren, C. Xie, X. Sun, Q. Zhang, and D. Yan, "Parameter identification of a lithium-ion battery based on the improved 
recursive least square algorithm," IET Power Electron., vol. 13, no. 12, pp. 2523-2530, 2020, doi: 10.1049/iet-pel.2019.1589.

C. Zhang, L. Y. Wang, X. Li, W. Chen, G. G. Yin, and J. Jiang, "Robust and Adaptive Estimation of State of Charge for Lithium-Ion Batteries," IEEE Trans. Ind. Electron., vol. 62, no. 8, pp. 4948-4957, 2015, doi: 10.1109/TIE.2015.2403796.

[13] A. I. Stroe, D. I. Stroe, M. Swierczynski, R. Teodorescu, and S. K. Kær, "Lithium-Ion battery dynamic model for wide range of operating conditions," Proc. - 2017 Int. Conf. Optim. Electr. Electron. Equipment, OPTIM 20172017 Intl Aegean Conf. Electr. Mach. Power Electron. ACEMP 2017, pp. 660-666, 2017, doi: 10.1109/OPTIM.2017.7975044.

[14] R. Xiong, F. Sun, Z. Chen, and H. He, “A data-driven multiscale extended Kalman filtering based parameter and state estimation approach of lithium-ion olymer battery in electric vehicles," Appl. Energy, vol. 113, pp. 463-476, 2014, doi: 10.1016/j.apenergy.2013.07.061.

[15] R. Xiong, S. Member, Y. Zhang, and S. Member, "A DoubleScale, Particle-Filtering, Energy State Prediction Algorithm for Lithium-Ion Batteries," IEEE Trans. Ind. Electron., vol. 65, no. 2, pp. 1526-1538, 2018.

[16] R. Xiong, F. Sun, H. He, and T. D. Nguyen, “A data-driven adaptive state of charge and power capability joint estimator of lithium-ion polymer battery used in electric vehicles," Energy, vol. 63, pp. 295-308, 2013, doi: 10.1016/j.energy.2013.10.027.

[17] V. H. Duong, H. A. Bastawrous, K. C. Lim, K. W. See, P. Zhang, and S. X. Dou, "Online state of charge and model parameters estimation of the $\mathrm{LiFePO} 4$ battery in electric vehicles using multiple adaptive forgetting factors recursive least-squares," J. Power Sources, vol. 296, pp. 215-224, 2015, doi: 10.1016/j.jpowsour.2015.07.041.

[18] X. Du et al., "An Information Appraisal Procedure Endows Reliable Online Parameter Identification to Lithium-ion Battery Model," IEEE Trans. Ind. Electron., vol. 0046, no. c, pp. 1-1, 2021, doi: 10.1109/tie.2021.3091920.

[19] X. Sun, J. Ji, B. Ren, C. Xie, and D. Yan, “Adaptive forgetting factor recursive least square algorithm for online identification of equivalent circuit model parameters of a lithium-ion battery," Energies, vol. 12, no. 11, 2019, doi: 10.3390/en12122242.

[20] T. Kim, Y. Wang, Z. Sahinoglu, T. Wada, S. Hara, and W. Qiao, "A Rayleigh Quotient-Based Recursive Total-Least-Squares Online Maximum Capacity Estimation for Lithium-Ion Batteries," IEEE Trans. Energy Convers., vol. 30, no. 3, pp. 842-851, 2015, doi: 10.1109/TEC.2015.2424673.

[21] Z. Wei, C. Zou, F. Leng, B. H. Soong, and K. J. Tseng, "Online model identification and state-of-charge estimate for lithium-ion battery with a recursive total least squares-based observer," IEEE Trans. Ind. Electron., vol. 65, no. 2, pp. 1336-1346, 2018, doi: 10.1109/TIE.2017.2736480.

[22] Z. Wei, G. Dong, X. Zhang, J. Pou, Z. Quan, and H. He, "NoiseImmune Model Identification and State-of-Charge Estimation for Lithium-Ion Battery Using Bilinear Parameterization," IEEE Trans. Ind. Electron., vol. 68, no. 1, pp. 312-323, 2021, doi: 10.1109/TIE.2019.2962429.

[23] C. Elisei-iliescu, C. Paleologu, J. Benesty, and C. Stanciu,
"Recursive Least-Squares Algorithms for the Identification of Low-Rank Systems,” vol. 27, no. 5, pp. 903-918, 2019.

[24] M. Chen and G. A. Rincón-Mora, "Accurate electrical battery model capable of predicting runtime and I-V performance," IEEE Trans. Energy Convers., vol. 21, no. 2, pp. 504-511, 2006, doi: 10.1109/TEC.2006.874229.

[25] W. Waag, S. Käbitz, and D. U. Sauer, "Experimental investigation of the lithium-ion battery impedance characteristic at various conditions and aging states and its influence on the application," Appl. Energy, vol. 102, pp. 885-897, 2013, doi: 10.1016/j.apenergy.2012.09.030.

\section{Biographical notes}

Xing-Hao Du, born in 1998, is currently pursuing the M.S. degree with the College of Electrical Engineering, Sichuan University, China. He received his bachelor degree from Civil Aviation Flight University of China, Deyang, China, in 2020. His research interests include lithium-ion batteries modeling, state-of-charge estimation and battery management systems.

Tel: +86-136-18087804; E-mail: duxinghao@stu.scu.edu.cn

Jin-Hao Meng, born in 1988, is currently an associate researcher in Sichuan University, Chengdu, China. He received the M.S. degree in control theory and control engineering and the Ph.D. degree in electrical engineering from Northwestern Polytechnical University (NPU), Xi'an, China, in 2013 and 2019, respectively. E-mail: jinhao@scu.edu.cn

Kai-Long Liu, is currently is a Research Fellow with the Warwick Manufacturing Group, University of Warwick, U.K. He received the B.Eng. degree in electrical engineering and the M.Sc. degree in control theory and control engineering from Shanghai University, China, in 2011 and 2014, respectively, and the Ph.D. degree in electrical engineering from the Energy, Power and Intelligent Control Group, Queen's University Belfast, Belfast, U.K., in 2018. E-mail:kliu02@qub.ac.uk

Ying-Min Zhang, born in 1974, is currently a Professor with the College of Electrical Engineering, Sichuan University, China. She received the M.S. and the Ph.D. degrees in electrical engineering from Sichuan University, Chengdu, China, in 1998 and 2013, respectively.

E-mail: zhangyingmin@scu.edu.cn

Shun-Li Wang, born in 1985, is currently a Professor with the School of Information Engineering, Southwest University of Science and Technology, China. He received the M.S. and the Ph.D. degrees from Southwest University of Science and Technology, China, in 2012 and 2018, respectively.

E-mail: wangshunli@swust.edu.cn

Ji-Chang Peng, born in 1988, is currently a lecturer in Nanjing Institute of Technology, Nanjing, China. He received the B.S. degree in electrical engineering from North China University of Water Resources and Electric Power, China, in 2010, and the M.S. degree in control theory and control engineering and the Ph.D. degree in electrical engineering, both from Northwestern 
Polytechnical University, China, in 2013 and 2019, respectively.

E-mail: linkpjc@gmail.com

Tian-Qi Liu, born in 1962, is currently a Professor with the College of Electrical Engineering, Sichuan University, China. She received the B.S. and the M.S. degrees from Sichuan University, China, in 1982 and 1986, respectively, and the Ph.D. degree from Chongqing University, China, in 1996, all in electrical engineering.

E-mail: tqliu@scu.edu.cn 